Article

\title{
Genomic Analysis of a Newly Isolated Acidithiobacillus ferridurans JAGS Strain Reveals Its Adaptation to Acid Mine Drainage
}

\author{
Jinjin Chen ${ }^{1,+} \oplus$, Yilan Liu ${ }^{1,+}$, Patrick Diep ${ }^{1}$ and Radhakrishnan Mahadevan ${ }^{1,2, *}$ \\ 1 Department of Chemical Engineering and Applied Chemistry, University of Toronto, \\ Toronto, ON M5S 3E5, Canada; jinjin.chen@utoronto.ca (J.C.); liu.yilan@utoronto.ca (Y.L.); \\ patrick.diep@mail.utoronto.ca (P.D.) \\ 2 Institute of Biomedical Engineering, University of Toronto, Toronto, ON M5S 3G9, Canada \\ * Correspondence: krishna.mahadevan@utoronto.ca \\ + Jinjin Chen and Yilan Liu contribute equally to this work.
}

Citation: Chen, J.; Liu, Y.; Diep, P.; Mahadevan, R. Genomic Analysis of a Newly Isolated Acidithiobacillus ferridurans JAGS Strain Reveals Its Adaptation to Acid Mine Drainage. Minerals 2021, 11, 74. https:// doi.org/10.3390/min11010074

Received: 20 November 2020 Accepted: 11 January 2021 Published: 13 January 2021

Publisher's Note: MDPI stays neutra with regard to jurisdictional claims in published maps and institutional affiliations.

Copyright: (c) 2021 by the authors. Licensee MDPI, Basel, Switzerland. This article is an open access article distributed under the terms and conditions of the Creative Commons Attribution (CC BY) license (https:/ / creativecommons.org/licenses/by/ $4.0 /)$.
Abstract: Acidithiobacillus ferridurans JAGS is a newly isolated acidophile from an acid mine drainage (AMD). The genome of isolate JAGS was sequenced and compared with eight other published genomes of Acidithiobacillus. The pairwise mutation distance (Mash) and average nucleotide identity (ANI) revealed that isolate JAGS had a close evolutionary relationship with $A$. ferridurans JCM18981, but whole-genome alignment showed that it had higher similarity in genomic structure with $A$. ferrooxidans species. Pan-genome analysis revealed that nine genomes were comprised of 4601 protein coding sequences, of which $43 \%$ were core genes (1982) and $23 \%$ were unique genes (1064). A. ferridurans species had more unique genes (205-246) than A. ferrooxidans species (21-234). Functional gene categorizations showed that $A$. ferridurans strains had a higher portion of genes involved in energy production and conversion while $A$. ferrooxidans had more for inorganic ion transport and metabolism. A high abundance of $k d p$, mer and ars genes, as well as mobile genetic elements, was found in isolate JAGS, which might contribute to its resistance to harsh environments. These findings expand our understanding of the evolutionary adaptation of Acidithiobacillus and indicate that $A$. ferridurans JAGS is a promising candidate for biomining and AMD biotreatment applications.

Keywords: Acidithiobacillus; acid mine drainage; biomining; comparative genomics

\section{Introduction}

With continually increasing concerns about acid mine drainage (AMD) contamination and the depletion of high-grade ores, innovative and sustainable methods to recover heavy metals from tailings and AMD as well as to treat AMD pollution are urgently needed [1]. Pyrometallurgical and hydrometallurgical routes are the conventional methods for metal recovery, but they are environmentally unsustainable, with a high cost in terms of operating on low-grade ores [2,3]. Though many techniques have been applied for AMD management, such as neutralization, adsorption, oxygen barriers, bactericides and so on, most of those options are unsustainable and unaffordable [4]. Compared with conventional and other emerging reprocessing techniques, bioleaching is considered as a simple, highly efficient, safe, low-cost, more easily managed and eco-friendly technique to facilitate sustainable mining and prevent AMD [3]. Bioleaching facilitates metal mobilization from solid metal sulfides into their water-soluble forms by different microorganisms via direct and indirect bioleaching [5]. Direct bioleaching can be summarized as:

$$
\mathrm{MeS}+2 \mathrm{O}_{2} \rightarrow \mathrm{MeSO}_{4}
$$


while indirect bioleaching can be described as:

$$
\mathrm{MeS}+\mathrm{Fe}_{2}\left(\mathrm{SO}_{4}\right)_{3} \rightarrow \mathrm{MeSO}_{4}+2 \mathrm{Fe}_{2} \mathrm{SO}_{4}+\mathrm{S}^{0}
$$

Microorganisms take part in and accelerate the oxidation of mineral sulfide to sulfate or the reoxidation of ferrous iron to ferric iron [6]. Although many factors affect the bioleaching process such as temperature, $\mathrm{pH}$, dissolved oxygen, redox potential and formation of secondary minerals [7-9], indigenous bacteria play a crucial role in effective bioleaching [10]. Therefore, an increasing research effort has been placed on discovering and characterizing indigenous robust microbes that are resistant to high metal concentrations in extremely acidic environments [11,12].

Acidithiobacillus is a group of Gram-negative, chemoautotrophic, acidophilic aerobes which dominates in all types of extremely acidic habitats, suggesting its tremendous potential in the bioleaching process [13]. Genome sequencing and analysis of this genus, revealing its adaption to harsh environments, will help us to better understand its mechanisms and provide insights for future genetic engineering strategies to make bioleaching more efficient and versatile. Li et al. [14] analyzed and validated genomic information from this genus, including seven species and 37 strains, and revealed that Acidithiobacillus spp. recruited and consolidated novel functionalities via horizontal gene transfer, gene duplication and purifying selection to cope with challenging environments. Presently, there are 10 species reported in this genus: A. ferrooxidans, A. ferridurans, A. ferrivorans, A. ferrianus, A. ferriphilus, A. albertensis, A. caldus, A. thiooxidans, A. sulfuriphilus and A. cuprithermicus. The first five species were reported to generate energy by oxidizing ferrous iron, sulfur and hydrogen. Since soluble ferric iron produced from this ferrous iron oxidation can serve as a powerful oxidant to accelerate the dissolution of sulfidic minerals to release target metals, Acidithiobacillus species such as A. ferrooxidans have drawn focused attention [7]. Since the 1940s, more than 500 isolates of $A$. ferrooxidans have been reported and whole-genome sequencing has been performed for nine isolates. The strain A. ferrooxidans ATCC23270 was chosen as the model strain in this genus because of its extensive description in the literature and its wide usage [15]. It can oxidize ferrous iron, reduced inorganic sulfur compounds (RISCs) and hydrogen to generate energy as ATP. Additionally, it can fix atmospheric carbon dioxide and nitrogen as nutrition sources [16]. A. ferrooxidans has been successfully applied to recover metals such as copper, nickel, zinc, arsenic and uranium from low-grade ores, sewage sludge and contaminated sediments [13].

A. ferridurans is a species reclassified from A. ferrooxidans by Hedrich and Johnson [17] in 2013 because the species' DNA-DNA hybridization (63\%) was lower than the threshold value $(70 \%)$ used to delineate species. Moreover, when comparing A. ferrooxidans ATCC23270 with A. ferridurans JCM18981 (formerly called A. ferrooxidans ATCC33020), the latter showed better resistance to lower $\mathrm{pH}$ and higher concentrations of $\mathrm{Fe}^{2+}, \mathrm{Ni}^{2+}$ and $\mathrm{Mg}^{2+}[17,18]$. Thus, the genus A. ferridurans might be a good candidate chassis for industrial applications in the field of biomining and bioremediation. For instance, a newly isolated A. ferridurans SBU-SH2 was used for flask and column bioleaching from lowgrade uranium ore, which generated $96 \%$ and $95.5 \%$ uranium extraction in 7 and 26 days, respectively $[19,20]$. However, at present, only one whole-genome sequence of $A$. ferridurans species, A. ferridurans JCM18981 isolated from uranium drainage water in Japan, is available [21]. The limited genome information of $A$. ferridurans species hinders our understanding of the mechanism and evolutionary history underpinning its unique metal and acid resistance.

In our previous study, a dominant strain belonging to the Acidithiobacillus genus was found based on $16 \mathrm{~S}$ rRNA gene sequence analysis, which took up $92.6 \%$ of the enriched culture from acid mine drainage (AMD) in Sudbury, Canada [22]. After whole-genome sequencing and assembly, we reclassified and named it as A. ferridurans JAGS based on simple 16S rRNA gene and ANIb analyses and announced its genome [23]. To better ununderstand this strain and provide useful data for future research, a detailed genomic analysis was further performed in this study. First, a side-by-side comparison of iron, nickel 
and low-pH tolerance between $A$. ferridurans JAGS and A. ferrooxidans ATCC23270 was conducted. To reveal the genetic traits associated with heavy metal and acid resistance in isolate JAGS, its genomic data were compared with the reported genomes of Acidithiobacillus strains. A pan-genome analysis was further conducted on these genomes to explore the metabolic features leading to the diversity of physico-biochemical traits. Functional genes and pathways responsible for heavy metal and acid resistance were analyzed and compared. A mobile genetic element analysis further suggested that gene transfers among these strains likely enabled adaptation to challenging environments. The insights gained in this study enhanced our understanding of the mechanism and evolutionary history of heavy metal and acid resistance in $A$. ferridurans and we suggest possible approaches for engineering A. ferridurans as a microbial chassis for biomining processes.

\section{Materials and Methods}

\subsection{Culture Media, Phenotypic and Growth Observations}

The strain A. ferridurans JAGS was isolated from acidic mine drainage in our previous study [22]. It was the dominant species and made up $92.6 \%$ of the enriched culture, based on 16S rRNA gene sequence analysis. The strain A. ferrooxidans ATCC23270 was purchased from American Type Culture Collection (ATCC). The phenotypic features of isolated JAGS were observed on a light microscope (Nikon Eclipse E400, Nikon, Melville, NY, USA) and a scanning electron microscope (FEI XL30 SEM, Philips, Eindhoven, Holland), separately. The strain JAGS was cultured with either $9 \mathrm{~K}-\mathrm{Fe}^{2+}(160 \mathrm{mM}$ ferrous iron, $\mathrm{pH} 2.0)$ or $9 \mathrm{~K}-\mathrm{S}^{0}$ $(0.5 \%$ elemental sulfur, $\mathrm{pH} 3.0)$ at $30^{\circ} \mathrm{C}$ with shaking at $180 \mathrm{rpm}$ or with $2: 2$ solid medium in an incubator at $30^{\circ} \mathrm{C}[24]$.

The abilities to tolerate elevated concentrations of ferrous iron and nickel and low $\mathrm{pH}$ were tested in a side-by-side comparison between $A$. ferridurans JAGS and A. ferrooxidans ATCC23270. Cultures grown in the $9 \mathrm{~K}-\mathrm{Fe}^{2+}$ medium were inoculated at a ratio of $20 \%$ into the same medium with $\mathrm{Fe}^{2+}$ (200 or $320 \mathrm{mM}$ ), $\mathrm{Ni}^{2+}(100$ or $200 \mathrm{mM}$ ) or at pH 1.5 and then incubated at $30{ }^{\circ} \mathrm{C}$ for $22 \mathrm{~h}$. The $\mathrm{Fe}^{2+}$ concentration was tested by the colorimetric ferrozine-based assay [25] and the ferrous iron oxidation rate was calculated by using consumed $\mathrm{Fe}^{2+}$ divided by its initial concentration, as in a previous study [26].

The growth features of $A$. ferridurans JAGS in $9 \mathrm{~K}-\mathrm{Fe}^{2+}$ or $9 \mathrm{~K}-\mathrm{S}^{0}$ medium were further monitored by detecting $\mathrm{pH}$, iron or sulfate concentrations and cell numbers during incubation by removing samples at intervals. The $\mathrm{pH}$ value was detected using a $\mathrm{pH}$ meter (Thermo Scientific ${ }^{\circledR}$, Orion Star A211, Waltham, MA, USA). Ferrous and ferric iron concentrations were examined by the colorimetric ferrozine-based assay [25]. Sulfate was detected using a turbidimetric method [27]. Three cell-counting methods were tested: direct cell counting, optical density $\left(\mathrm{OD}_{600}\right)$ measurements and plate counting. For the direct cell-counting method, samples were taken from media and cell numbers were estimated using a hemocytometer (Hausser Scientific, Horsham, PA, USA). For the $\mathrm{OD}_{600}$ method, cells were harvested, washed twice with a basal salt buffer $\left(4.5 \mathrm{~g} / \mathrm{L}\left(\mathrm{NH}_{4}\right)_{2} \mathrm{SO}_{4}, 0.15 \mathrm{~g} / \mathrm{L}\right.$ $\mathrm{KCl}$ and $0.75 \mathrm{~g} / \mathrm{L} \mathrm{MgSO}_{4} \cdot 7 \mathrm{H}_{2} \mathrm{O}$ ) and resuspended in $6 \%$ betaine prior to measurements. The plate counting was carried out by spreading proper diluted samples on 2:2 solid plates and colonies were counted after 7-10 days.

\subsection{Comparative Genomics}

Details of $A$. ferridurans JAGS genomic DNA extraction, sequencing, assembly and annotation are described in our previous paper [23]. The complete genome sequence of the isolate JAGS contains 2,933,811 bp with a GC (guanine-cytosine) content of 58.6\%. The Similar Genome Finder service on the PATRIC website was used with the default parameters to find the other similar Acidithiobacillus genomes published and to calculate their Mash/MinHash distances with isolate JAGS [28]. For these genomes, average nucleotide identities based on BLAST (ANIb) and MUMmer (ANIm) were calculated in JSpeciesWS [29]. Genome alignment among four whole-genome sequences was achieved 
using progressiveMauve within PATRIC [30]. Genes related to acid stress and metal resistance were analyzed using PATRIC and created with BioRender (https:/ / biorender.com).

\subsection{Pan-Genome Analysis}

NCBI PGAP [31] was used to predict coding sequences for $A$. ferridurans JAGS and 8 other Acidithiobacillus genomes, and these amino acid sequences were used as the input for the Bacterial Pan-genome Analysis tool (BPGA ver. 1.2) to estimate core and pan genomes using the USEARCH program (ver. 9.0) available in BPGA, with a 50\% cut-off of sequence identity [32]. The empirical power law equation $f(n)=a \times n^{\alpha}$ and the exponential equation $\mathrm{f} 1(\mathrm{n})=\mathrm{c} \times \mathrm{e}^{(\mathrm{d} . \mathrm{n})}$ were used for extrapolation of the pan and core genome curves, respectively. Core, accessory and unique genes defined in USEARCH were mapped into various cluster of orthologous group (COG) categories and Kyoto Encyclopedia of Genes and Genomes (KEGG) pathways. The EggNOG (ver. 5.0.0) program [33] with default parameters was further used to cluster genes into functionally related groups and to analyze metabolic pathways.

\subsection{Prediction of Mobile Genetic Elements}

Insertion sequences (ISs) and transposases (Tn) distributed over the 9 Acidithiobacillus genomes were predicted and classified using the ISFinder platform with manual inspection of search hits (E-value $\leq 10^{-5}$ ) [34]. IslandViewer (ver. 4), which has integrated the three most accurate and complementary genomic islands (GIs) prediction tools, IslandPathDIMOB, SIGI-HMM and IslandPick [35], was applied for the computational identification of putative GIs. In addition, the web tool CRISPRFinder was mainly used to identify the Clustered Regularly Interspaced Short Palindromic Repeats-Cas protein (CRISPRCas) array.

\section{Results and Discussion}

\subsection{Phenotypic and Growth Features}

The genus of Acidithiobacillus is widely distributed in natural environments such as acid mine drainage (AMD) settings. We isolated an Acidithiobacillus strain from an AMD sample collected from Sudbury, Canada, and named it $A$. ferridurans JAGS in our previous study [23]. Orange-brown colonies of isolate JAGS formed on solid media, taking the shape of dots after 10 days of incubation (Figure 1A). The cells of isolate JAGS collected from $9 \mathrm{~K}-\mathrm{Fe}^{2+}$ liquid media showed single and paired rods, approximately $0.5-1.5 \mu \mathrm{m}$ long and $0.3 \mu \mathrm{m}$ wide (Figure $1 \mathrm{~B}$ ), which is slightly smaller than the reported $A$. ferrooxidans that is 1-2 $\mu \mathrm{m}$ long and 0.3-0.6 $\mu \mathrm{m}$ wide.


Figure 1. Differentiation of Acidithiobacillus ferridurans JAGS from closely related species. (A) Colony morphologies of A. ferridurans JAGS observed under $40 \times$ optical microscopy for both top and bottom images. (B) Cell morphology observed under SEM. (C) Ferrous iron oxidation rates of A. ferridurans JAGS and A. ferrooxidans ATCC23270 under different pressures of Fe200 $\left(200 \mathrm{mM} \mathrm{Fe}^{2+}\right)$, Fe320 (320 $\left.\mathrm{mM} \mathrm{Fe}^{2+}\right)$, Ni100 $\left(100 \mathrm{mM} \mathrm{Ni}^{2+}\right), \mathrm{Ni} 200\left(200 \mathrm{mM} \mathrm{Ni}^{2+}\right)$ and $\mathrm{pH} 1.5 .{ }^{* *}$ indicates $p<0.01$. 
The species of $A$. ferridurans was reported to have a notably higher tolerance to many metals such as $\mathrm{Fe}^{2+}, \mathrm{Ni}^{2+}$ and $\mathrm{Mg}^{2+}$ and lower $\mathrm{pH}$ when compared with other Acidithiobacillus species $[17,18]$. In addition, there is $0.5-1 \% \mathrm{Ni}$ existing in the pyrrhotite tailings of Sudbury [36]. Therefore, we compared the $\mathrm{Fe}^{2+}$ oxidation rate (\%) between A. ferridurans JAGS and A. ferrooxidans ATCC23270 under elevated concentrations of $\mathrm{Fe}^{2+}$ and $\mathrm{Ni}^{2+}$ and low $\mathrm{pH}$ pressures (Figure 1C). The results showed that isolate JAGS had higher $\mathrm{Fe}^{2+}$ oxidation rates compared with ATCC23270 under high concentrations of $\mathrm{Fe}^{2+}$ and low $\mathrm{pH}$ values but similar levels of $\mathrm{Fe}^{2+}$ oxidation rates under high concentrations of $\mathrm{Ni}^{2+}$. These results indicated the adaptation of $A$. ferridurans JAGS to the acid mine drainage in Sudbury and suggested that it might be a great candidate as the ferrous oxidizer in low-pH bioleaching.

The growth features of $A$. ferridurans JAGS in culture media with $9 \mathrm{~K}-\mathrm{Fe}^{2+}$ and $9 \mathrm{~K}-\mathrm{S}^{0}$ were investigated (Supplementary Material Figure S1). Figure S1A,B present a standard curve of $\mathrm{OD}_{600}$ versus the cell count obtained by plate counting. When $\mathrm{OD}_{600}=1$, we estimated that there were $8.8 \times 10^{9}$ cells $/ \mathrm{mL}$ of $A$. ferridurans JAGS, which is slightly higher than the reported number of $A$. ferrooxidans $\left(8.3 \times 10^{9}\right.$ cells $\left./ \mathrm{mL}\right)$ [37]. This may be due to the smaller cell size of isolate JAGS that causes it to absorb less light in the cuvette. We noted that counting cells of isolate JAGS was difficult due to its very low cell density in the lag phase and the interference of precipitate formation in the exponential phase. Therefore, monitoring growth required indirect tracking via changes in the $\mathrm{pH}$ and electron donor concentrations, but this was corroborated with data from $\mathrm{OD}_{600}$ measurements and the plate count method. The growth behavior of isolate JAGS in 9K-Fe ${ }^{2+}$ (Figure S1C) and 9K-S0 culture media (Figure S1D) shared similarities with what was reported for A. ferrooxidans ATCC 23270 [38,39], which indicates a close relationship between the two species. However, the cell numbers examined by $\mathrm{OD}_{600}$ were much lower than those obtained by the plate count method, which is likely due to the cells lost during the process of precipitate removal prior to $\mathrm{OD}_{600}$ measurements.

\subsection{Genomic Features}

To better understand the isolated strain $A$. ferridurans JAGS, its genome was sequenced (GenBank: CP044411) and analyzed. The genome of $A$. ferridurans JAGS is a single circular chromosome comprising $2,933,811$ bases with a GC content of $58.56 \%$, which contains 3001 protein-coding sequences (CDSs), 46 tRNAs and 6 rRNAs [23]. The genomic features of A. ferridurans JAGS are quite similar to those of $A$. ferridurans JCM18981, which are 2,921,399 bases with 58.4\% GC content, containing 3026 CDSs, 47 tRNAs and 6 rRNAs.

To explore the relationship of $A$. ferridurans JAGS with other Acidithiobacillus species, the Similar Genome Finder service from PATRIC was used to find similar genomes with isolate JAGS as the reference. There were eight Acidithiobacillus genomes found (Table 1). These strains were collected from different environments but mainly from acid mine waters. Their genomes varied in size from 2.7 to $3.2 \mathrm{Mb}$, with total CDS numbers ranging from 2634 to 3179. For the Mash/MinHash distances, A. ferridurans JCM18981 and A. ferrooxidans IO-2C showed the closest distance with $A$. ferridurans JAGS compared to the other six strains, which suggests that the IO-2C strain might be incorrectly classified. Based on the average nucleotide identity (ANI) relatedness analysis, it appears that the strains JAGS, JCM18981 and IO-2C are all A. ferridurans species as they shared ANI values $>98 \%$ with each other (Table 1 and Table S1), which is larger than the reported threshold of $\geq 96 \%$ for classification [14].

Genome alignment among the complete-genome sequences of $A$. ferridurans JAGS, A. ferridurans JCM18981, A. ferrooxidans ATCC53993 and A. ferrooxidans ATCC23270 was performed using progressiveMauve [24] and is shown in Figure 2. Surprisingly, the genomic arrangement of $A$. ferridurans JAGS had better co-linearity with $A$. ferrooxidans ATCC53993 and A. ferrooxidans ATCC23270 than with $A$. ferridurans JCM18981. The samecolor blocks suggest high conservation of gene orders among multiple genomes that are likely inherited through vertical transfer, while $A$. ferridurans JCM18981 had a large 
number of gene rearrangements, insertions and/or deletions. This result indicates multiple recombination events and a rich evolutionary history of $A$. ferridurans species.

Table 1. General features and genomic comparison (pairwise mutation (Mash) distance, average nucleotide identity (ANI)) between A. ferridurans JAGS and selected representatives.

\begin{tabular}{|c|c|c|c|c|c|c|c|c|c|}
\hline $\begin{array}{l}\text { Genome } \\
\text { Name }\end{array}$ & Geographic Origin & Contigs & $\begin{array}{l}\text { Genome } \\
\text { Size }\end{array}$ & GC\% & $\begin{array}{l}\text { No. of } \\
\text { CDS }\end{array}$ & $\begin{array}{l}\text { BioProject } \\
\text { Accession }\end{array}$ & $\begin{array}{c}\text { Mash } \\
\text { Distance }\end{array}$ & ANIb & ANIm \\
\hline $\begin{array}{l}\text { A. ferridurans } \\
\text { JAGS }\end{array}$ & $\begin{array}{c}\text { Acid mine drainage, } \\
\text { Canada }\end{array}$ & 1 & $2,933,811$ & 58.6 & 3001 & PRJNA573091 & - & - & 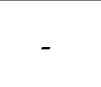 \\
\hline $\begin{array}{l}\text { A. ferridurans } \\
\text { JCM18981 }\end{array}$ & $\begin{array}{c}\text { Uranium mine } \\
\text { drainage water, } \\
\text { Japan }\end{array}$ & 1 & 2,921,399 & 58.4 & 3026 & PRJDB7175 & 0.0090 & 99.13 & 99.66 \\
\hline $\begin{array}{l}\text { A. ferrooxidans } \\
\text { IO-2C }\end{array}$ & Acid seep soil, USA & 23 & $2,716,894$ & 58.7 & 2634 & PRJNA432283 & 0.0136 & 98.69 & 99.23 \\
\hline $\begin{array}{l}\text { A. ferrooxidans } \\
\text { ATCC53993 }\end{array}$ & - & 1 & $2,885,038$ & 58.9 & 2826 & PRJNA16689 & 0.0446 & 94.98 & 95.51 \\
\hline $\begin{array}{l}\text { A. ferrooxidans } \\
\text { RVS1 }\end{array}$ & $\begin{array}{l}\text { Andacollo gold } \\
\text { mining area, } \\
\text { Argentina }\end{array}$ & 49 & $2,826,311$ & 58.8 & 2705 & PRJNA499028 & 0.0463 & 94.68 & 95.33 \\
\hline $\begin{array}{l}\text { A. ferrooxidans } \\
\text { CCM4253 }\end{array}$ & $\begin{array}{c}\text { Mine waters, Czech } \\
\text { Republic }\end{array}$ & 15 & $3,196,562$ & 58.6 & 3059 & PRJNA475418 & 0.0480 & 94.70 & 95.32 \\
\hline $\begin{array}{l}\text { A. ferrooxidans } \\
\text { YQH-1 }\end{array}$ & $\begin{array}{c}\text { Wudalianchi } \\
\text { volcano water, China }\end{array}$ & 96 & $3,111,222$ & 58.6 & 3089 & PRJNA294114 & 0.0482 & 94.71 & 95.34 \\
\hline $\begin{array}{l}\text { A. ferrooxidans } \\
\text { Hel18 }\end{array}$ & Flue dust & 123 & $3,109,160$ & 58.6 & 3179 & PRJNA308169 & 0.0484 & 94.69 & 95.34 \\
\hline $\begin{array}{l}\text { A. ferrooxidans } \\
\text { ATCC23270 }\end{array}$ & $\begin{array}{l}\text { bituminous coal } \\
\text { mine effluent }\end{array}$ & 1 & $2,982,397$ & 58.8 & 3147 & PRJNA53 & 0.0493 & 94.76 & 95.37 \\
\hline
\end{tabular}

Note: Average nucleotide identity (ANI) based on BLAST+ (ANIb) and MUMmer (ANIm).

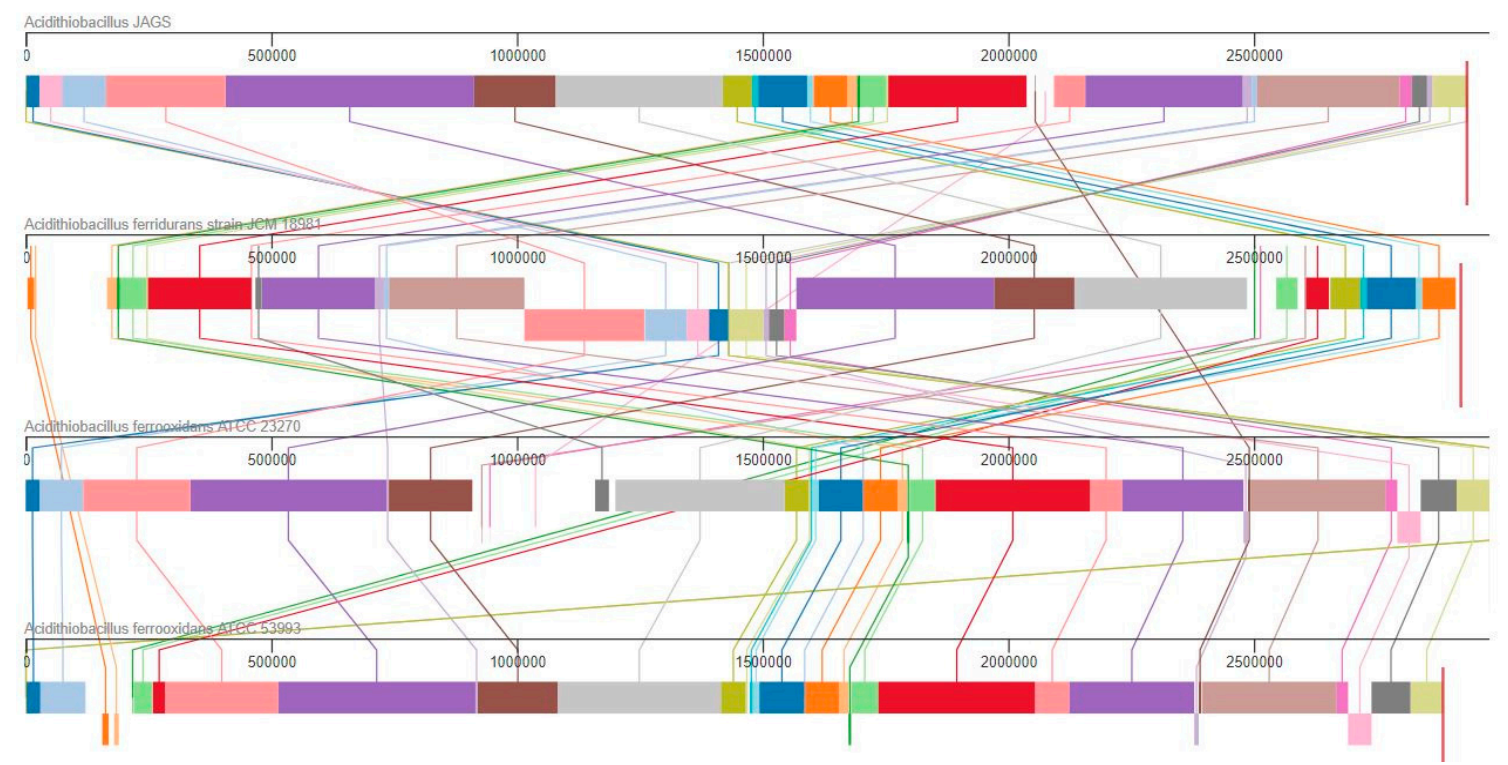

Figure 2. Whole-genome alignment of A. ferridurans JAGS, A.ferridurans JCM18981, A. ferrooxidans ATCC53993 and A. ferrooxidans ATCC23270. Locally collinear blocks (LCBs) identified by Mauve are color-coded; links between LCBs are indicated by the thin colored lines.

\subsection{Pan-Genome and Functional Gene Analysis}

Pan-genome analysis was carried out using the Bacterial Pan-genome Analysis (BPGA) tool to provide insights regarding genomic features, diversity and evolution [32]. It is well accepted that more than five genomes in a pan-genome analysis could provide suf- 
ficient data for extrapolation of the information for species [40]. In this study, genomes of $A$. ferridurans JAGS and eight other strains of $A$. ferridurans and $A$. ferrooxidans were used for the pan-genome analysis since we wanted to investigate the genetic diversity and ecological adaption of these two species. As shown in Figure 3A,B, according to the Heaps ${ }^{\prime}$ Law function $\left(f(n)=2819.13 n^{0.22}\right)$, the pan genome is open as the $\gamma$ was calculated as 0.22 , which means that the addition of new genomes will provide novel genes and it indicates evolutionary changes in these genomes [41]. The pan genome contains a total of 4601 genes, of which 1982 genes are in the core genome and 1064 genes are in the unique genome. The richness of unique genes in A. ferridurans JAGS, JCM18981, IO-2C and A. ferrooxidans ATCC23270 suggests that they may actively exchange genes with other genera to adapt to different environmental conditions.
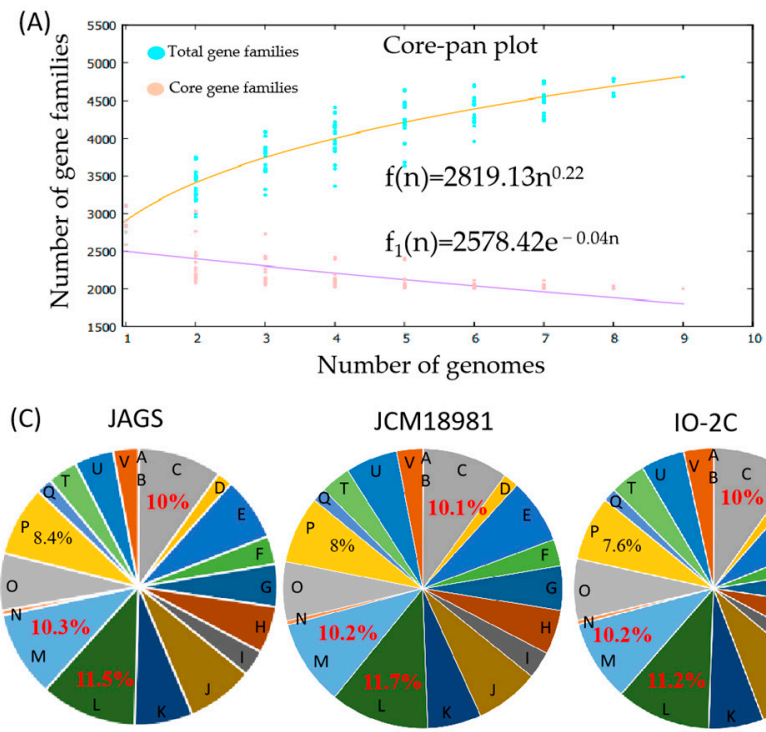

CCM4253

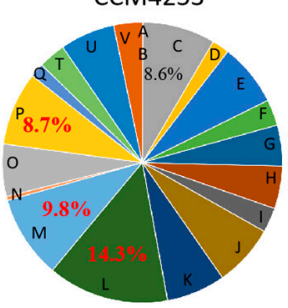

JCM18981

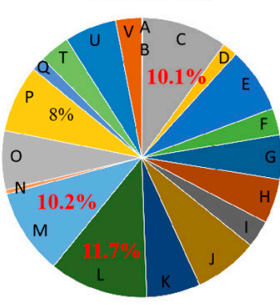

YQH-1

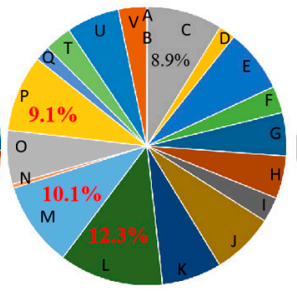

IO-2C

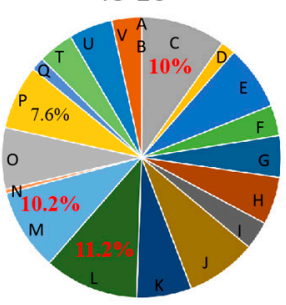

Hel18

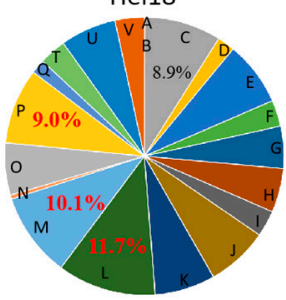

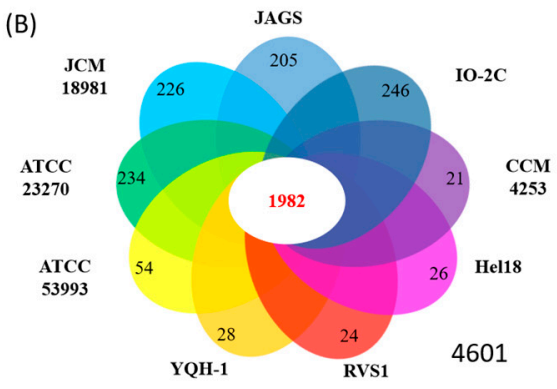

ATCC53993
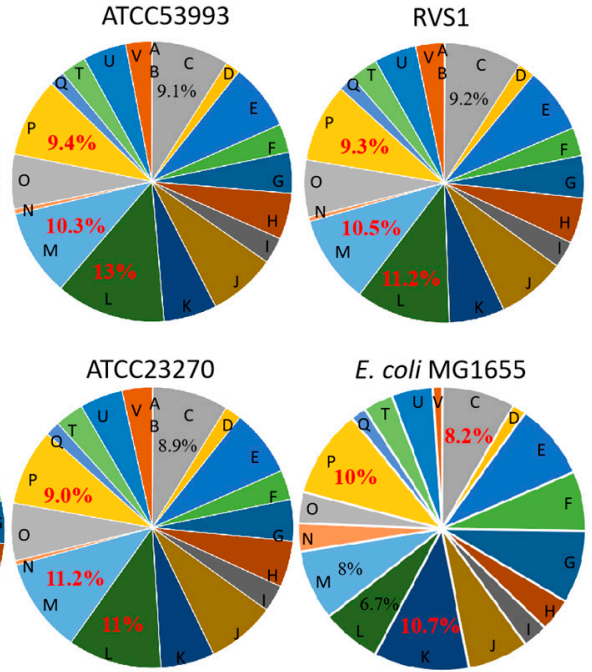

E. coli MG1655

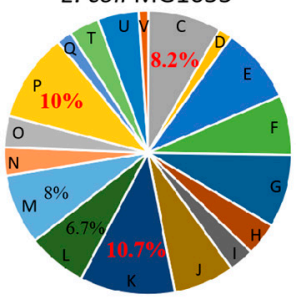

Figure 3. Pan-genome and EggNOG analysis. (A) Core-pan plot of studied Acidithiobacillus genomes; (B) Venn diagram of the pan genome; (C) pie charts of cluster of orthologous groups (COGs) of studied A. ferridurans and A. ferrooxidans strains and E. coli MG1655 (reference). Note: In Figure 3C, the numbers represent the percentage of each category. A, RNA processing and modification; B, Chromatin structure and dynamics; C, Energy production and conversion; D, Cell cycle control and mitosis; E, Amino acid metabolism and transport; F, Nucleotide metabolism and transport; G, Carbohydrate metabolism and transport; H, Coenzyme metabolism; I, Lipid metabolism; J, Translation; K, Transcription; L, Replication and repair; M, Cell wall/membrane/envelope biogenesis; N, Cell motility; O, Post-translational modification, protein turnover, chaperone functions; P, Inorganic ion transport and metabolism; Q, Secondary structure; T, Signal transduction; U, Intracellular trafficking and secretion; V, Defense mechanisms. The categories of " $\mathrm{R}$, General functional prediction only" and "S, Function unknown" were omitted.

The cluster of orthologous group (COG) distributions of the annotated genes for each studied Acidithiobacillus strain are illustrated in Figure 3C. These COGs fell into 20 COG classes, not including "General functional prediction only" and "Function unknown". Escherichia coli MG1655 was used as a reference, which showed that E. coli had the highest portion of genes corresponding to (K) Transcription, while Acidithiobacillus strains showed higher portions of functional genes related to (L) Replication, recombination and repair 
(10.7-14\%) and (M) Cell wall/membrane/envelope biogenesis (9.4-10.9\%). This was not surprising given that these categories of proteins have been reported to be necessary for acid and heavy metal resistance and, likely, long-term adaptation mechanisms to extreme environments [42]. When the two Acidithiobacillus species were compared, A. ferridurans had more genes associated with functions supporting $(C)$ Energy production and conversion $(9.7-9.9 \%)$, while A. ferrooxidans had more genes related to the function of $(\mathrm{P})$ Inorganic ion transport and metabolism (8.5-9.2\%).

\subsection{Genetic Mechanisms of Acid Stress and Metal Resistance.}

In response to acidic heavy metal stress, acidophiles have developed different genetic mechanisms to survive and they are described, which can be very complex $[43,44]$. The metabolic diversity and adaptive mechanisms of Acidithiobacillus spp. responding to extremely acidic environments have been reviewed [45] and are beyond the scope of this study. Here, we focused on five major mechanisms (Figure 4A) for acid and heavy metal resistance in representative Acidithiobacillus strains by analyzing related gene clusters: (1) a membrane barrier created by outer membrane proteins (Omp40) and hopanoids; (2) maintenance of a membrane potential by influx of potassium and sequestration of metal ions intra-/extracellularly; (3) active removal by antiporters or exporters; (4) decarboxylation and detoxification; and (5) DNA and protein repair systems.

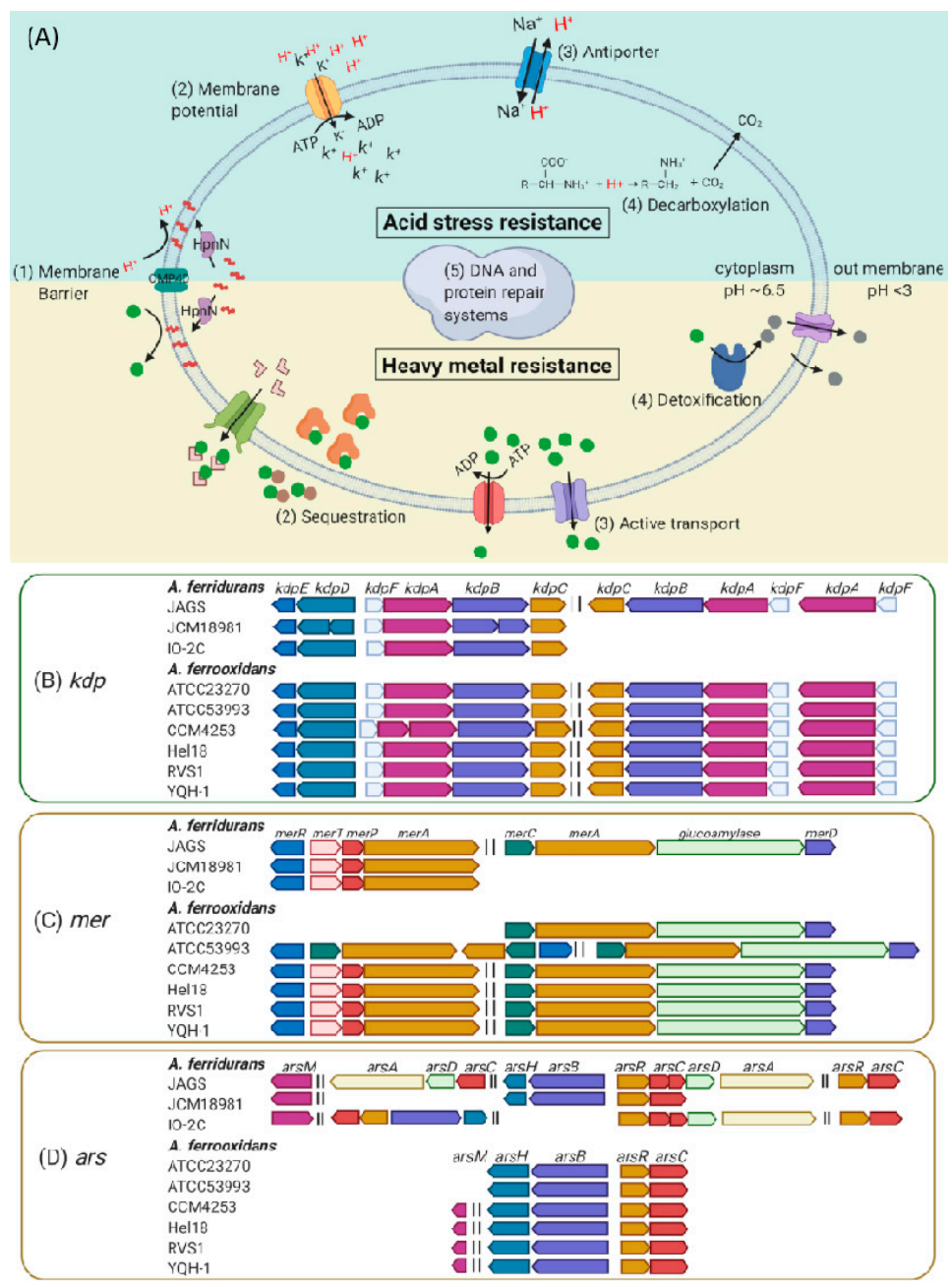

Figure 4. Overview of adaptive strategies for acid and heavy metal resistance. Potential resistance mechanisms (A) and comparisons of operons for $k d p(\mathbf{B})$, mer (C) and ars (D) genes among Acidithiobacillus strains. Kdp, a high-affinity $\mathrm{K}^{+}$transport system; mer, $\mathrm{Hg}^{2+}$-resistant genes; ars, $\mathrm{As}^{2+/ 3+}$-resistant genes. 
The mechanisms responsible for acid stress resistance are complex. We analyzed relevant genes in A. ferridurans JAGS and listed them in Table S2 (Supplementary Materials). OMP40 (Gene ID: F6A13_00370) was discovered and it was previously reported as an anionic porin in the outer membrane to restrict the influx of protons in A. ferrooxidans [46]. A number of genes coding for hopanoid-synthesis proteins were identified in the isolate JAGS genome, including a glycosyltransferase (HpnB, Gene ID: F6A13_01630) and a cluster for hopanoid-associated proteins, a squalene-hopene cyclase and a squalene synthase (HpnMHNKJIAG-Sch-Sqs, Gene IDs: F6A13_09105-09160). Hopanoid is an important type of bacterial lipid that can alter membrane fluidity and permeability to restrict $\mathrm{H}^{+}$influx. It was reported to be synthesized from squalene by SHC, HpnG and other proteins enriched in the outer membrane by a transporter $\mathrm{HpnN}$, although the functions of some factors are still unknown [47].

The $\mathrm{Na}^{+} / \mathrm{H}^{+}$antiporter (Gene IDs: F6A13_09475, 04755) can export excess protons by coupling the uptake of $\mathrm{Na}^{+}$, while decarboxylases (SpeA, PanD and Psd; Gene IDs: F6A13_06090, 14595, 04545) will consume excess protons in the cytoplasm. For most of the proteins, the interspecies identities ranged from $94 \%$ to $98 \%$, while intraspecies identities were $100 \%$. Interestingly, we found the A. ferridurans JAGS genome to process three $k d p$ clusters ( $k d p E D F A B C$, Gene IDs: F6A13_09590-09605; $k d p F A B C$, Gene IDs: 11020-11005; kdpFA, GeneIDs: 11030-11025), similar to other A. ferrooxidans strains. However, A. ferridurans JCM18981 and IO-2C only had one $k d p$ cluster (Figure 4B). The $k d p$ clusters code for a high-affinity $\mathrm{K}^{+}$transport system, which could generate a reversed membrane potential through the active influx of $\mathrm{K}^{+}$to cope with acid resistance [48]. In Figure S2 (Supplementary Material), a neighbor-joining (NJ) phylogenetic tree is constructed based on the kdpA protein sequences. Even though $A$. ferridurans JAGS had a similar pattern of $k d p$ clusters to $A$. ferrooxidans species (Figure 4B), its $k d p A$ (I) showed higher sequence identity with the other $A$. ferridurans strains, suggesting that $k d p$ clusters can be acquired more than once in these genomes and have redundancy.

We also tried to find genes involved in heavy metal resistance pathways in $A$. ferridurans JAGS (Figure 4 and Table S2). In total, eight genes (Gene IDs: F6A13_04860, 04905, $04925,04945,08625,10865,10890,11740)$ were predicted to code for p-type ATPases to transport $\mathrm{Pb}^{2+}, \mathrm{Cd}^{2+}, \mathrm{Zn}^{2+}, \mathrm{Hg}^{2+}$ and $\mathrm{Cu}^{2+}$ [49]. Similar numbers of these genes were detected in ATCC53993 (8) and JCM18981 (9), while only 5-6 genes were found in other strains. Some of these genes are highly similar, such as the genes F6A13_10865 and F6A13_04945, which indicates gene duplication within a genome. There are several other clusters belonging to the Resistance-nodulation-division (RND) transporter system, in the $c z c$ or $z n u$ families, which corresponds to $\mathrm{Ni}^{2+}, \mathrm{Mn}^{2+}, \mathrm{Fe}^{2+/ 3+}, \mathrm{Mo}^{2+}, \mathrm{Co}^{2+}, \mathrm{Cd}^{2+}$ and $\mathrm{Zn}^{2+}$ and CorAC for $\mathrm{Mg}^{2+} / \mathrm{Co}^{2+}$ export. We also noticed that these Acidithiobacillus strains possessed two types and several copies of $c z c A B C$ clusters located at different sites, suggesting that $c z c$ clusters may be acquired more than once from different origins. Overall, this pattern of redundancy of resistance clusters in Acidithiobacillus suggests that it is part of the adaptive strategy for survival in acidic heavy metal conditions.

We also found genes coding for proteins related to mercury $\left(\mathrm{Hg}^{2+}\right)$ and arsenic $\left(\mathrm{As}^{2+/ 3+}\right)$ resistance. The operons we found for $\mathrm{Hg}^{2+}$ resistance in Acidithiobacillus strains are summarized in Figure $4 \mathrm{C}$. There were two subgroups of mer clusters detected, merRTPA and merCAD. A. ferridurans JAGS and A. ferrooxidans CCM4253, Hel18, RVS1 and YQH-1 possessed both mer clusters, while $A$. ferridurans JCM18981 and IO-2C lacked the merCAD cluster. An intact merRTPA cluster was not detected in A. ferrooxidans ATCC23270 and ATCC53993, although ATCC53993 had two copies of merR. The merA in the merCAD cluster exhibited $100 \%$ identity in all selected strains, suggesting that merCAD might come from the same donor. Therefore, we further investigated the genetic context of the cluster merCAD and found it adjacent to $k d p C B A F A F$ clusters. The DNA sequences of the $k d p C B A F A F-$ merCAD clusters $(11,906 \mathrm{bp})$ in $A$. ferridurans JAGS share $100 \%$ identity and $100 \%$ coverage with $A$. ferrooxidans ATCC53993, ATCC23270, RVS1 and CCM4253 and 100\% identity with less coverage for $A$. ferrooxidans Hel18 and YQH-1, possibly due to incomplete sequencing. 
This indicates that the $k d p C B A F A F-m e r C A D$ clusters might come from a same donor via a horizontal gene transfer (HGT) event [14]. Two predicted mobile element proteins (Gene IDs: F6A13_10980, 10985) were found upstream of the $k d p C B A F A F-m e r C A D$ clusters in isolate JAGS, supporting the HGT hypothesis.

Annotation of arsenic resistance clusters included $\operatorname{ars} H B R C D A$, $\operatorname{ars} H R B C, \operatorname{ars} C D A$, $\operatorname{ars} R C$ and $\operatorname{ars} M$ in the studied genomes. A. ferridurans JAGS possessed all of these clusters, while the arsCDA and arsRC clusters were absent in A. ferridurans JCM18981 and the studied A. ferrooxidans strains. The largest abundance of ars clusters in isolate JAGS compared with other strains might contribute to its dominance in our metal-rich mine drainage sample. Besides, the analysis of ars $C$ protein sequences further suggested that the ars clusters $\operatorname{ars} H B R C D A$, ars $H B R C$ and $\operatorname{ars} R C$ are likely acquired from different donors during evolution. Since it was also reported that gene copy number alterations can benefit microorganisms' survival under selective pressure [50], we hypothesize that $A$. ferridurans JAGS might have gained ars clusters from other species during adaption to the metal-rich environments.

In summary, compared to the other Acidithiobacillus strains that we studied, A. ferridurans JAGS had several genes and considerable redundancy that likely contributes to its acid and heavy metal resistance, which highlights its strong potential for usage in biomining processes, especially for cinnabar ( $\mathrm{HgS}$ ) or arsenopyrite (FeAsS) tailings. Additionally, we speculate that $A$. ferridurans JAGS might be an intermediate species between $A$. ferrooxidans and $A$. ferridurans based on the evidence of the gene cluster types and genomic structure (Figure 4) and the genome alignment result (Figure 2).

\subsection{Mobile Genetic Elements Analysis}

Mobile genetic elements (MGEs) play a great role in genome plasticity and evolution, shaping both genes and genomes to respond to drastic changes in environmental conditions [51]. MGEs, including insertion sequences (ISs) and genomic islands (GIs), are listed in Table 2. The number of ISs per strain ranged from 38 (A. ferridurans IO-2C) to 78 (A. ferridurans JAGS and A. ferridurans JCM18981), which might be due to the genome assembly level. High similarity regarding IS type was observed in all studied strains: IS1595, IS21, IS3, ISL3 and Tn families, which were the most common IS families. IS3 was the most abundant family. However, closer inspection demonstrated several differences. For instance, A. ferridurans JAGS has a much higher number of IS1595 when compared with other species. The nine genomes harbored 19-26 GIs ranging from 4 to $158 \mathrm{~kb}$ in size, representing many versatile gene pools. Several ISs, such as IS110 and IS66, were presented in the predicted GIs, suggesting that these putative GIs were likely acquired by horizontal gene transfer. In addition, GIs carrying mercury resistance genes (merRTPA) were found in all A. ferridurans species but not in the model strains A. ferrooxidans ATCC23270 and A. ferrooxidans ATCC53993, which might provide A. ferridurans with an adaptive advantage in mercury-rich environments.

Furthermore, we examined the clustered regularly interspaced short palindromic repeats (CRISPR) systems in all studied genomes using the CRISPRCasFinder [52]. Interestingly, A. ferrooxidans ATCC23270 was the only strain with a predicted CRISPR system. One unique type IV Cas cluster (csf4-1-2-3) and five spacers presented in the vicinity were found and presumed to function in conjunction with other CRISPR arrays [53]. 
Table 2. The prediction of mobile genetic elements including insertion sequences (ISs) and genomic islands (GIs) of the Acidithiobacillus strains studied.

\begin{tabular}{|c|c|c|c|c|c|c|c|}
\hline \multicolumn{8}{|c|}{ A. The Putative Insertion Sequences } \\
\hline IS Family & JAGS & JCM18981 & IO-2C & ATCC53993 & CCM4253 & ATCC23270 & RVS1 \\
\hline IS110 & 3 & 3 & 1 & 4 & 4 & 3 & 1 \\
\hline IS1182 & 0 & 1 & 0 & 1 & 0 & 0 & - \\
\hline IS1595 & 15 & 1 & 3 & 6 & 0 & 1 & - \\
\hline IS1634 & 2 & 6 & 4 & 2 & 1 & 6 & - \\
\hline IS200/IS605 & 3 & 2 & 0 & 1 & 0 & 2 & - \\
\hline IS21 & 7 & 17 & 3 & 9 & 6 & 17 & 1 \\
\hline IS256 & 7 & 7 & 0 & 2 & 2 & 7 & - \\
\hline IS3 & 21 & 23 & 14 & 21 & 22 & 23 & - \\
\hline IS481 & 1 & 0 & 0 & 1 & 1 & 0 & - \\
\hline IS5 & 1 & 1 & 2 & 12 & 2 & 1 & - \\
\hline IS66 & 2 & 2 & 2 & 2 & 2 & 2 & - \\
\hline IS7 & 1 & 1 & 1 & 1 & 1 & 1 & - \\
\hline ISL3 & 8 & 8 & 7 & 7 & 8 & 8 & - \\
\hline $\operatorname{Tn}$ & 7 & 6 & 1 & 5 & 3 & 6 & - \\
\hline In total & 78 & 78 & 38 & 74 & 52 & 77 & - \\
\hline \multicolumn{8}{|c|}{ B. The Predicted Genomic Islands } \\
\hline GI No. & 22 & 22 & 20 & 21 & 26 & 21 & 19 \\
\hline Size range $(\mathrm{Kb})$ & $4-42$ & $4-46$ & $4-28$ & $4-158$ & $4-63$ & $4-25$ & $4-35$ \\
\hline
\end{tabular}

Note: Since RVS1, Hel18 and YQH-1 have many contigs, ISFinder and IslandViewer cannot predict ISs and GIs exactly, respectively.

\section{Conclusions}

In the present study, we provide the growth characteristics and genomic insights of a newly isolated strain, A. ferridurans JAGS. The growth features of isolate JAGS in $9 \mathrm{~K}-\mathrm{Fe}^{2+}$ and $9 \mathrm{~K}-\mathrm{S}^{0}$ liquid media are similar to the A. ferrooxidans type strain ATCC23270, but it shows a higher oxidation rate under elevated concentrations of $\mathrm{Fe}^{2+}$ and low $\mathrm{pH}$. Genomic comparison and pan-genome analysis among nine strains of two species of $A$. ferridurans and A. ferrooxidans revealed obvious genetic differences between the two species. A. ferridurans JAGS showed a closer evolutionary relationship with other $A$. ferridurans species but a higher similarity of genomic structure with the $A$. ferrooxidans strains. This suggests that A. ferridurans JAGS might be an intermediary strain. Investigations of gene clusters ( $k d p$, mer and ars) and mobile genetic elements indicated that there have been frequent gene transfers between their genomes during evolution. The high abundance of acid and metal resistance genes found in A. ferridurans JAGS points to its unique abilities to survive in harsh mining environments, which highlights its strong potential for applications in biomining processes. Further transcriptomic and proteomic analyses are required to find the exact genes, proteins and possible mechanisms that lead to the increased resistance of the isolate JAGS strain.

Supplementary Materials: The following are available online at https://www.mdpi.com/2075-1 63X/11/1/74/s1, Figure S1: Growth features of $A$. ferridurans JAGS. (A) Standard curve of optical density $\left(\mathrm{OD}_{600}\right)$ versus cell numbers obtained by plate count method. (B) Colonies on 2:2 solid medium for cell count. (C) Growth on $9 \mathrm{~K}-\mathrm{Fe}^{2+}$ medium, (C1) pH value; (C2) Fe oxidation; (C3) cell numbers by $\mathrm{OD}_{600}$; (C4) cell numbers by plate count method. (D) Growth on $9 \mathrm{~K}-\mathrm{S}^{0}$ medium, (D1) $\mathrm{pH}$ value; (D2) sulfur oxidation; (D3) cell count by $\mathrm{OD}_{600}$; (D4) cell numbers by plate count method. Figure S2: Neighbor-joining (NJ) phylogenetic tree of the kdpA protein sequences derived from nine Acidithiobacillus strains. Bootstrap values indicated at each node are based on a total of 500 bootstrap replicates. Table S1: Average nucleotide identity (ANI) (\%) based on whole-genome alignments among Acidithiobacillus strains by JSpeciesWS. Table S2: Genes predicted to be involved in acid and heavy metal tolerance in A. ferridurans JAGS. 
Author Contributions: Conceptualization, J.C. and R.M.; methodology, J.C. and Y.L.; software, formal analysis and writing —original draft preparation, J.C. and Y.L.; writing—review and editing, P.D. and R.M.; supervision, R.M.; project administration, R.M.; funding acquisition, R.M. All authors have read and agreed to the published version of the manuscript.

Funding: This work was funded through the Elements of Biomining Grant from the Province of Ontario through the ORF Research Excellence funding program.

Institutional Review Board Statement: Not applicable.

Informed Consent Statement: Not applicable.

Data Availability Statement: Not applicable.

Acknowledgments: Vale is acknowledged for providing access to sample tailings at their Sudbury, ON, Canada, operations.

Conflicts of Interest: The authors declare no conflict of interest.

\section{References}

1. Igarashi, T.; Herrera, P.S.; Uchiyama, H.; Miyamae, H.; Iyatomi, N.; Hashimoto, K.; Tabelin, C.B. The two-step neutralization ferrite-formation process for sustainable acid mine drainage treatment: Removal of copper, zinc and arsenic, and the influence of coexisting ions on ferritization. Sci. Total Environ. 2020, 715, 136877. [CrossRef]

2. Thao, N.T.; Tsuji, S.; Jeon, S.; Park, I.; Tabelin, C.B.; Ito, M.; Hiroyoshi, N. Redox potential-dependent chalcopyrite leaching in acidic ferric chloride solutions: Leaching experiments. Hydrometallurgy 2020, 194, 105299. [CrossRef]

3. Baniasadi, M.; Vakilchap, F.; Bahaloo-Horeh, N.; Mousavi, S.M.; Farnaud, S. Advances in bioleaching as a sustainable method for metal recovery from e-waste: A review. J. Ind. Eng. Chem. 2019, 76, 75-90. [CrossRef]

4. Park, I.; Tabelin, C.B.; Jeon, S.; Li, X.; Seno, K.; Ito, M.; Hiroyoshi, N. A review of recent strategies for acid mine drainage prevention and mine tailings recycling. Chemosphere 2019, 219, 588-606. [CrossRef] [PubMed]

5. Vera, M.; Schippers, A.; Sand, W. Progress in bioleaching: Fundamentals and mechanisms of bacterial metal sulfide oxidationpart A. Appl. Microbiol. Biotechnol. 2013, 97, 7529-7541. [CrossRef] [PubMed]

6. Bosecker, K. Bioleaching: Metal solubilization by microorganisms. FEMS Microbiol. Rev. 1997, 20, 591-604. [CrossRef]

7. Tabelin, C.B.; Corpuz, R.D.; Igarashi, T.; Villacorte-Tabelin, M.; Alorro, R.D.; Yoo, K.; Raval, S.; Ito, M.; Hiroyoshi, N. Acid mine drainage formation and arsenic mobility under strongly acidic conditions: Importance of soluble phases, iron oxyhydroxides/oxides and nature of oxidation layer on pyrite. J. Hazard. Mater. 2020, 399, 122844. [CrossRef]

8. Rasoulnia, P.; Barthen, R.; Lakaniemi, A.M. A critical review of bioleaching of rare earth elements: The mechanisms and effect of process parameters. Crit. Rev. Environ. Sci. Technol. 2020, 1-50. [CrossRef]

9. Park, I.; Tabelin, C.B.; Magaribuchi, K.; Seno, K.; Ito, M.; Hiroyoshi, N. Suppression of the release of arsenic from arsenopyrite by carrier-microencapsulation using Ti-catechol complex. J. Hazard. Mater. 2018, 344, 322-332. [CrossRef]

10. Wu, C.; Jiang, M.; Hsieh, L.; Cai, Y.; Shen, Y.; Wang, H.; Lin, Q.; Shen, C.; Hu, B.; Lou, L. Feasibility of bioleaching of heavy metals from sediment with indigenous bacteria using agricultural sulfur soil conditioners. Sci. Total Environ. 2020, 703, 134812. [CrossRef]

11. Camargo, F.P.; do Prado, P.F.; Tonello, P.S.; Dos Santos, A.C.A.; Duarte, I.C.S. Bioleaching of toxic metals from sewage sludge by co-inoculation of Acidithiobacillus and the biosurfactant-producing yeast Meyerozyma guilliermondii. J. Environ. Manag. 2018, 211, 28-35. [CrossRef] [PubMed]

12. Giese, E.C.; Carpen, H.L.; Bertolino, L.C.; Schneider, C.L. Characterization and bioleaching of nickel laterite ore using Bacillus subtilis strain. Biotechnol. Prog. 2019, 35, e2860. [CrossRef] [PubMed]

13. Zhang, S.; Yan, L.; Xing, W.; Chen, P.; Zhang, Y.; Wang, W. Acidithiobacillus ferrooxidans and its potential application. Extremophiles 2018, 22, 563-579. [CrossRef] [PubMed]

14. Li, L.; Liu, Z.; Meng, D.; Liu, X.; Li, X.; Zhang, M.; Tao, J.; Gu, Y.; Zhong, S.; Yin, H. Comparative genomic analysis reveals the distribution, organization, and evolution of metal resistance genes in the Genus Acidithiobacillus. Appl. Environ. Microbiol. 2019, 85, e02153-18. [CrossRef] [PubMed]

15. Valdés, J.; Pedroso, I.; Quatrini, R.; Dodson, R.J.; Tettelin, H.; Blake, R.; Eisen, J.A.; Holmes, D.S. Acidithiobacillus ferrooxidans metabolism: From genome sequence to industrial applications. BMC Genom. 2008, 9, 597. [CrossRef] [PubMed]

16. Campodonico, M.A.; Vaisman, D.; Castro, J.F.; Razmilic, V.; Mercado, F.; Andrews, B.A.; Feist, A.M.; Asenjo, J.A. Acidithiobacillus ferrooxidans's comprehensive model driven analysis of the electron transfer metabolism and synthetic strain design for biomining applications. Metab. Eng. Commun. 2016, 3, 84-96. [CrossRef]

17. Hedrich, S.; Johnson, D.B. Acidithiobacillus ferridurans sp. nov., an acidophilic iron-, sulfur-and hydrogen-metabolizing chemolithotrophic gammaproteobacterium. Int. J. Syst. Evol. Microbiol. 2013, 63, 4018-4025.

18. Falagán, C.; Moya-Beltrán, A.; Castro, M.; Quatrini, R.; Johnson, D.B. Acidithiobacillus sulfuriphilus sp. nov.: An extremely acidophilic sulfur-oxidizing chemolithotroph isolated from a neutral $\mathrm{pH}$ environment. Int. J. Syst. Evol. Microbiol. 2019, $69,2907-2913$. 
19. Jalali, F.; Fakhari, J.; Zolfaghari, A. Response surface modeling for lab-scale column bioleaching of low-grade uranium ore using a new isolated strain of Acidithiobacillus Ferridurans. Hydrometallurgy 2019, 185, 194-203. [CrossRef]

20. Jalali, F.; Fakhar, J.; Zolfaghari, A. On using a new strain of Acidithiobacillus ferridurans for bioleaching of low-grade uranium. Sep. Sci. Technol. 2020, 55, 994-1004. [CrossRef]

21. Miyauchi, T.; Kouzuma, A.; Abe, T.; Watanabe, K. Complete genome sequence of Acidithiobacillus ferridurans JCM 18981. Microbiol. Resour Announc 2018, 7, e01028-18. [CrossRef]

22. Garg, S. Abiotic and Biotic Leaching Characteristics of Pyrrhotite Tailings from the Sudbury, Ontario Area. Ph.D. Thesis, University of Toronto, Toronto, ON, Canada, 2017.

23. Chen, J.; Liu, Y.; Diep, P.; Jo, A.; Nesbø, C.; Edwards, E.; Papangelakis, V.; Mahadevan, R. Complete genome sequence of Acidithiobacillus ferridurans JAGS, isolated from acidic mine drainage. Microbiol. Resour. Announc. 2020, 9, 9. [CrossRef]

24. Wang, H.; Liu, X.; Liu, S.; Yu, Y.; Lin, J.; Lin, J.; Pang, X.; Zhao, J. Development of a markerless gene replacement system for Acidithiobacillus ferrooxidans and construction of a pfkB mutant. Appl. Environ. Microbiol. 2012, 78, 1826-1835. [CrossRef] [PubMed]

25. Stookey, L.L. Ferrozine-A new spectrophotometric reagent for iron. Anal. Chem. 1970, 42, 779-781. [CrossRef]

26. Daoud, J.; Karamanev, D. Formation of jarosite during $\mathrm{Fe}^{2+}$ oxidation by Acidithiobacillus ferrooxidans. Miner. Eng. 2006, 19, 960-967. [CrossRef]

27. Kolmert, Å.; Wikström, P.; Hallberg, K.B. A fast and simple turbidimetric method for the determination of sulfate in sulfatereducing bacterial cultures. J. Microbiol. Methods 2000, 41, 179-184. [CrossRef]

28. Wattam, A.R.; Davis, J.J.; Assaf, R.; Boisvert, S.; Brettin, T.; Bun, C.; Conrad, N.; Dietrich, E.M.; Disz, T.; Gabbard, J.L. Improvements to PATRIC, the all-bacterial bioinformatics database and analysis resource center. Nucleic Acids Res. 2017, 45, D535-D542. [CrossRef] [PubMed]

29. Richter, M.; Rosselló-Móra, R.; Oliver Glöckner, F.; Peplies, J. JSpeciesWS: A web server for prokaryotic species circumscription based on pairwise genome comparison. Bioinformatics 2016, 32, 929-931. [CrossRef]

30. Darling, A.E.; Mau, B.; Perna, N.T. progressiveMauve: Multiple genome alignment with gene gain, loss and rearrangement. PloS ONE 2010, 5, e11147. [CrossRef]

31. Tatusova, T.; DiCuccio, M.; Badretdin, A.; Chetvernin, V.; Nawrocki, E.P.; Zaslavsky, L.; Lomsadze, A.; Pruitt, K.D.; Borodovsky, M.; Ostell, J. NCBI prokaryotic genome annotation pipeline. Nucleic Acids Res. 2016, 44, 6614-6624. [CrossRef]

32. Chaudhari, N.M.; Gupta, V.K.; Dutta, C. BPGA-an ultra-fast pan-genome analysis pipeline. Sci. Rep. 2016, 6, 1-10. [CrossRef]

33. Huerta-Cepas, J.; Szklarczyk, D.; Heller, D.; Hernández-Plaza, A.; Forslund, S.K.; Cook, H.; Mende, D.R.; Letunic, I.; Rattei, T.; Jensen, L.J. eggNOG 5.0: A hierarchical, functionally and phylogenetically annotated orthology resource based on 5090 organisms and 2502 viruses. Nucleic Acids Res. 2019, 47, D309-D314. [CrossRef]

34. Siguier, P.; Pérochon, J.; Lestrade, L.; Mahillon, J.; Chandler, M. ISfinder: The reference centre for bacterial insertion sequences. Nucleic Acids Res. 2006, 34, D32-D36. [CrossRef] [PubMed]

35. Bertelli, C.; Laird, M.R.; Williams, K.P.; Group, S.F.U.R.C.; Lau, B.Y.; Hoad, G.; Winsor, G.L.; Brinkman, F.S. IslandViewer 4 : Expanded prediction of genomic islands for larger-scale datasets. Nucleic Acids Res. 2017, 45, W30-W35. [CrossRef] [PubMed]

36. Rezaei, S.; Liu, F.; Marcuson, S.; Muinonen, M.; Lakshmanan, V.; Sridhar, R.; Barati, M. Canadian pyrrhotite treatment: The history, inventory and potential for tailings processing. Can. Metall. Q. 2017, 56, 410-417. [CrossRef]

37. Kernan, T.; Majumdar, S.; Li, X.; Guan, J.; West, A.C.; Banta, S. Engineering the iron-oxidizing chemolithoautotroph Acidithiobacillus ferrooxidans for biochemical production. Biotechnol. Bioeng. 2016, 113, 189-197. [CrossRef]

38. Beard, S.D.; Paradela, A.D.; Albar, J.P.D.; Jerez, C.A.D. Growth of Acidithiobacillus ferrooxidans ATCC 23270 in thiosulfate under oxygen-limiting conditions generates extracellular sulfur globules by means of a secreted tetrathionate hydrolase. Front. Microbiol. 2011, 2, 79. [CrossRef]

39. Shen, X.; Hu, H.; Peng, H.; Wang, W.; Zhang, X. Comparative genomic analysis of four representative plant growth-promoting rhizobacteria in Pseudomonas. BMC Genom. 2013, 14, 271. [CrossRef]

40. Vernikos, G.; Medini, D.; Riley, D.R.; Tettelin, H. Ten years of pan-genome analyses. Curr. Opin. Microbiol. 2015, $23,148-154$. [CrossRef]

41. Hinger, I.; Ansorge, R.; Mussmann, M.; Romano, S. Phylogenomic analyses of members of the widespread marine heterotrophic genus Pseudovibrio suggest distinct evolutionary trajectories and a novel genus, Polycladidibacter gen. nov. Appl. Environ. Microbiol. 2020, 86, 86. [CrossRef]

42. Mi, S.; Song, J.; Lin, J.; Che, Y.; Zheng, H.; Lin, J. Complete genome of Leptospirillum ferriphilum ML-04 provides insight into its physiology and environmental adaptation. J. Microbiol. 2011, 49, 890-901. [CrossRef]

43. Mirete, S.; Morgante, V.; González-Pastor, J.E. Acidophiles: Diversity and mechanisms of adaptation to acidic environments. In Adaption of Microbial Life to Environmental Extremes; Springer: Cham, Switzerland, 2017; pp. 227-251.

44. Vergara, E.; Neira, G.; González, C.; Cortez, D.; Dopson, M.; Holmes, D.S. Evolution of predicted acid resistance mechanisms in the extremely acidophilic Leptospirillum genus. Genes 2020, 11, 389. [CrossRef]

45. Zhang, X.; Liu, X.; Liang, Y.; Fan, F.; Zhang, X.; Yin, H. Metabolic diversity and adaptive mechanisms of iron-and/or sulfuroxidizing autotrophic acidophiles in extremely acidic environments. Environ. Microbiol. Rep. 2016, 8, 738-751. [CrossRef] [PubMed]

46. Guiliani, N.; Jerez, C.A. Molecular Cloning, Sequencing, and Expression ofomp-40, the gene coding for the major outer membrane protein from the acidophilic bacterium Thiobacillus ferrooxidans. Appl. Environ. Microbiol. 2000, 66, 2318-2324. [CrossRef] [PubMed] 
47. Belin, B.J.; Busset, N.; Giraud, E.; Molinaro, A.; Silipo, A.; Newman, D.K. Hopanoid lipids: From membranes to plant-bacteria interactions. Nat. Rev. Microbiol. 2018, 16, 304. [CrossRef] [PubMed]

48. Nanatani, K.; Shijuku, T.; Takano, Y.; Zulkifli, L.; Yamazaki, T.; Tominaga, A.; Souma, S.; Onai, K.; Morishita, M.; Ishiura, M. Comparative analysis of $\mathrm{kdp}$ and ktr mutants reveals distinct roles of the potassium transporters in the model cyanobacterium Synechocystis sp. strain PCC 6803. J. Bacteriol. 2015, 197, 676-687. [CrossRef] [PubMed]

49. Nies, D.H. Efflux-mediated heavy metal resistance in prokaryotes. FEMS Microbiol. Rev. 2003, 27, 313-339. [CrossRef]

50. Tang, Y.-C.; Amon, A. Gene copy-number alterations: A cost-benefit analysis. Cell 2013, 152, 394-405. [CrossRef]

51. Shapiro, J. Mobile Genetic Elements; Elsevier: Amsterdam, The Netherlands, 2012.

52. Couvin, D.; Bernheim, A.; Toffano-Nioche, C.; Touchon, M.; Michalik, J.; Néron, B.; Rocha, E.P.; Vergnaud, G.; Gautheret, D.; Pourcel, C. CRISPRCasFinder, an update of CRISRFinder, includes a portable version, enhanced performance and integrates search for Cas proteins. Nucleic Acids Res. 2018, 46, W246-W251. [CrossRef]

53. Makarova, K.S.; Haft, D.H.; Barrangou, R.; Brouns, S.J.; Charpentier, E.; Horvath, P.; Moineau, S.; Mojica, F.J.; Wolf, Y.I.; Yakunin, A.F. Evolution and classification of the CRISPR-Cas systems. Nat. Rev. Microbiol. 2011, 9, 467. [CrossRef] 